\title{
A characterization of $\operatorname{Ext}(G, \mathbb{Z})$ assuming $(V=L)$
}

\author{
by \\ Saharon Shelah (Jerusalem and New Brunswick, NJ) and \\ Lutz Strüngmann (Essen and Honolulu)
}

\begin{abstract}
We complete the characterization of $\operatorname{Ext}(G, \mathbb{Z})$ for any torsion-free abelian group $G$ assuming Gödel's axiom of constructibility plus there is no weakly compact cardinal. In particular, we prove in $(V=L)$ that, for a singular cardinal $\nu$ of uncountable cofinality which is less than the first weakly compact cardinal and for every sequence $\left(\nu_{p}: p \in \Pi\right)$ of cardinals satisfying $\nu_{p} \leq 2^{\nu}$ (where $\Pi$ is the set of all primes), there is a torsion-free abelian group $G$ of size $\nu$ such that $\nu_{p}$ equals the $p$-rank of $\operatorname{Ext}(G, \mathbb{Z})$ for every prime $p$ and $2^{\nu}$ is the torsion-free $\operatorname{rank}$ of $\operatorname{Ext}(G, \mathbb{Z})$.
\end{abstract}

1. Introduction. Since the first author solved the well known Whitehead problem in 1977 (see [Sh1], [Sh2]) the structure of $\operatorname{Ext}(G, \mathbb{Z})$ for torsionfree abelian groups $G$ has received much attention. Easy arguments show that $\operatorname{Ext}(G, \mathbb{Z})$ is always a divisible group for every torsion-free group $G$. Hence it is of the form

$$
\operatorname{Ext}(G, \mathbb{Z})=\bigoplus_{p \in \Pi} \mathbb{Z}\left(p^{\infty}\right)^{\left(\nu_{p}\right)} \oplus \mathbb{Q}^{\left(\nu_{0}\right)}
$$

for some cardinals $\nu_{p}, \nu_{0}(p \in \Pi)$ which are uniquely determined (and $\Pi$ is the set of all prime numbers). Thus, the obvious question that arises is which sequences $\left(\nu_{0}, \nu_{p}: p \in \Pi\right)$ can appear as the cardinal invariants of $\operatorname{Ext}(G, \mathbb{Z})$ for some (which) torsion-free abelian group? On the one hand, there are a few results about possible sequences $\left(\nu_{0}, \nu_{p}: p \in \Pi\right)$ provable in ZFC. For instance, the trivial sequence consisting of zero entries only can be realized by any free abelian group. On the other hand, assuming Gödel's constructible universe $(V=L)$ plus there is no weakly compact car-

2000 Mathematics Subject Classification: Primary 20K15, 20K20, 20K35, 20K40; Secondary 18E99, $20 \mathrm{~J} 05$.

The first author was supported by project No. I-706-54.6/2001 of the German-Israeli Foundation for Scientific Research \& Development. Number 873 in Shelah's list of publications. DFG.

The second author was supported by a grant from the German Research Foundation 
dinal it has been shown that almost all sequences (with natural restrictions) can be the cardinal invariants of $\operatorname{Ext}(G, \mathbb{Z})$ for some torsion-free abelian group $G$ whenever the size of the group $G$ is not a singular cardinal of uncountable cofinality (see Section 2 for details and [EkHu], [EkSh], [GrSh1], [GrSh2], [HiHuSh], [MeRoSh], [SaSh1], and [SaSh2] for references). However, the question of which sequences $\left(\nu_{0}, \nu_{p}: p \in \Pi\right)$ can occur is independent of ZFC. It is the purpose of this paper to deal with the remaining case, namely torsion-free abelian groups of cardinality $\nu$ where $\nu$ is singular of cofinality $\operatorname{cf}(\nu)>\aleph_{0}$. The idea is to use the construction principle from [MeRoSh] which holds under $(V=L)$ and to apply the main theorem from [MeRoSh] in our construction.

Our notation is standard and we write maps from the left. If $H$ is a pure subgroup of the abelian group $G$, then we shall write $H \subseteq_{*} G$. For further details on abelian groups we refer to $[\mathrm{Fu}]$ and for set-theoretic methods to [EkMe], [Je] or $[\mathrm{Ku}]$.

2. The structure of $\operatorname{Ext}(G, \mathbb{Z})$. In this section we recall the basic results on the structure of $\operatorname{Ext}(G, \mathbb{Z})$ for torsion-free $G$. It is easy to see that $\operatorname{Ext}(G, \mathbb{Z})$ is divisible for torsion-free $G$, hence it is of the form

$$
\operatorname{Ext}(G, \mathbb{Z})=\bigoplus_{p \in \Pi} \mathbb{Z}\left(p^{\infty}\right)^{\left(\nu_{p}\right)} \oplus \mathbb{Q}^{\left(\nu_{0}\right)}
$$

for some cardinals $\nu_{p}, \nu_{0}(p \in \Pi)$. Since the cardinals $\nu_{p}(p \in \Pi)$ and $\nu_{0}$ completely determine the structure of $\operatorname{Ext}(G, \mathbb{Z})$ we introduce the following terminology. We denote by $r_{0}^{\mathrm{e}}(G)$ the torsion-free rank $\nu_{0}$ of $\operatorname{Ext}(G, \mathbb{Z})$, which is the dimension of $\mathbb{Q} \otimes \operatorname{Ext}(G, \mathbb{Z})$, and by $r_{p}^{\mathrm{e}}(G)$ the $p$-rank $\nu_{p}$ of $\operatorname{Ext}(G, \mathbb{Z})$, which is the dimension of $\operatorname{Ext}(G, \mathbb{Z})[p]$ as a vector space over $\mathbb{Z} / p \mathbb{Z}$ for any prime number $p \in \Pi$. There are only a few results provable in ZFC when $G$ is uncountable, but assuming Gödel's universe an almost complete characterization is known (if there is no weakly compact cardinal). The aim of this paper is to fill the remaining gap.

We first justify our restriction to torsion-free $G$. Let $A$ be any abelian group and $t(A)$ its torsion subgroup. Then $\operatorname{Hom}(t(A), \mathbb{Z})=0$ and hence we obtain the short exact sequence

$$
0 \rightarrow \operatorname{Ext}(A / t(A), \mathbb{Z}) \rightarrow \operatorname{Ext}(A, \mathbb{Z}) \rightarrow \operatorname{Ext}(t(A), \mathbb{Z}) \rightarrow 0
$$

which must split since $\operatorname{Ext}(A / t(A), \mathbb{Z})$ is divisible. Thus

$$
\operatorname{Ext}(A, \mathbb{Z}) \cong \operatorname{Ext}(A / t(A), \mathbb{Z}) \oplus \operatorname{Ext}(t(A), \mathbb{Z}) .
$$

Since the structure of $\operatorname{Ext}(t(A), \mathbb{Z}) \cong \prod_{p \in \Pi} \operatorname{Hom}\left(A, \mathbb{Z}\left(p^{\infty}\right)\right)$ is well known in $\mathrm{ZFC}$ it is reasonable to assume that $A$ is torsion-free and, of course, non-free. Using Pontryagin's theorem one proves 
LEMMA 2.1. Suppose $G$ is a countable torsion-free group which is not free. Then $r_{0}^{\mathrm{e}}(G)=2^{\aleph_{0}}$.

Proof. See [EkMe, Theorem XII 4.1].

Similarly, for the $p$-ranks of $G$ we have the following result due to C. U. Jensen.

LEMMA 2.2. If $G$ is a countable torsion-free group, then for any prime $p$, either $r_{p}^{\mathrm{e}}(G)$ is finite or $2^{\aleph_{0}}$.

Proof. See [EkMe, Theorem XII 4.7].

This clarifies the structure of $\operatorname{Ext}(G, \mathbb{Z})$ for countable torsion-free groups $G$ since the existence of groups as in Lemmas 2.1 and 2.2 follows from Lemma 2.8 below. We now turn our attention to uncountable groups and assume Gödel's axiom of constructibility. The following is due to Hiller, Huber and Shelah.

Lemma 2.3. $(V=L)$ Suppose $G$ is a torsion-free non-free group and let $B$ be a subgroup of $A$ of minimum cardinality $\nu$ such that $A / B$ is free. Then $r_{0}^{\mathrm{e}}(G)=2^{\nu}$. In particular, $r_{0}^{\mathrm{e}}(G)$ is uncountable and $r_{0}^{\mathrm{e}}(G)=2^{|G|}$ if $G^{*}=\operatorname{Hom}(G, \mathbb{Z})=0$.

Proof. See [EkMe, Theorem XII 4.4, Corollary XII 4.5].

Note that the above lemma is not true in ZFC since for any countable divisible group $D$ it is consistent that there exists an uncountable torsion-free group $G$ with $\operatorname{Ext}(G, \mathbb{Z}) \cong D$, hence $r_{0}^{\mathrm{e}}(G)=1$ is possible by taking $D=\mathbb{Q}$ (see Shelah [Sh3]).

Again we turn to $p$-ranks. There is a useful characterization of $r_{p}^{\mathrm{e}}(G)$ using the exact sequence

$$
0 \rightarrow \mathbb{Z} \stackrel{p}{\rightarrow} \mathbb{Z} \rightarrow \mathbb{Z} / p \mathbb{Z} \rightarrow 0
$$

The induced sequence

$$
\operatorname{Hom}(G, \mathbb{Z}) \stackrel{\varphi^{p}}{\rightarrow} \operatorname{Hom}(G, \mathbb{Z} / p \mathbb{Z}) \rightarrow \operatorname{Ext}(G, \mathbb{Z}) \stackrel{p_{*}}{\rightarrow} \operatorname{Ext}(G, \mathbb{Z})
$$

shows that the dimension of

$$
\operatorname{Hom}(G, \mathbb{Z} / p \mathbb{Z}) / \operatorname{Hom}(G, \mathbb{Z}) \varphi^{p}
$$

as a vector space over $\mathbb{Z} / p \mathbb{Z}$ is exactly $r_{p}^{\mathrm{e}}(G)$.

The following result due to Mekler, Rosłanowski and Shelah shows that under the assumption of $(V=L)$ almost all possibilities for $r_{p}^{\mathrm{e}}(G)$ can appear if the group is of regular cardinality.

LEMMA 2.4. $(V=L)$ Let $\nu$ be an uncountable regular cardinal less than the first weakly compact cardinal. Suppose that $\left(\nu_{p}: p \in \Pi\right)$ is a sequence of cardinals such that for each $p, 0 \leq \nu_{p} \leq 2^{\nu}$. Then there is an almost-free group $G$ of cardinality $\nu$ such that $r_{0}^{\mathrm{e}}(G)=2^{\nu}$ and for all $p, r_{p}^{\mathrm{e}}(G)=\nu_{p}$. 
Proof. See [MeRoSh, Main Theorem 3.9].

On the other hand, if the cardinality of $G$ is singular, then the following holds, which was proved by Grossberg and Shelah.

LEMMA 2.5. If $\nu$ is a singular strong limit cardinal of cofinality $\omega$, then there is no torsion-free group $G$ of cardinality $\nu$ such that $r_{p}^{\mathrm{e}}(G)=\nu$ for any prime $p$.

Proof. See [GrSh1, Theorem 1.0].

Note that Lemma 2.4 shows that the restriction in Lemma 2.5 is the only restriction for singular strong limit cardinals $\nu$ of cofinality $\omega$. Namely, if $\varrho<\nu$ choose a regular cardinal $\varrho \leq \varrho^{\prime}<\nu$ and apply Lemma 2.4 to obtain a torsion-free group $G^{\prime}$ with $r_{p}^{\mathrm{e}}\left(G^{\prime}\right)=\varrho$ and $\left|G^{\prime}\right|=\varrho^{\prime}$. Since $\operatorname{Ext}(-, \mathbb{Z})$ is a multiplicative functor we can now easily get a torsion-free group $G$ from $G^{\prime}$ with $|G|=\nu$ and $r_{p}^{\mathrm{e}}(G)=\varrho$.

Also the case of weakly compact cardinality was dealt with in [SaSh1] by Sageev and Shelah.

LEMMA 2.6. If $G$ is a torsion-free group of weakly compact cardinality $\nu$ and $r_{p}^{\mathrm{e}}(G) \geq \nu$ for some prime $p$, then $r_{p}^{\mathrm{e}}(G)=2^{\nu}$.

Proof. See [SaSh1, Main Theorem].

The above results show that under the assumption of $(V=L)$ the structure of $\operatorname{Ext}(G, \mathbb{Z})$ for torsion-free groups $G$ of cardinality $\nu$ is clarified for all cardinals $\nu$ except when $\nu$ is singular but not of cofinality $\omega$. This will be the subject of the next section.

However, in a particular case, namely when $G^{*}=\operatorname{Hom}(G, \mathbb{Z})=0$, a complete characterization of $\operatorname{Ext}(G, \mathbb{Z})$ is known in Gödel's universe if there is no weakly compact cardinal. The following is due to Hiller-Huber-Shelah.

LEMMA 2.7. If $G$ is torsion-free such that $\operatorname{Hom}(G, \mathbb{Z})=0$, then for all primes $p, r_{p}^{\mathrm{e}}(G)$ is finite or of the form $2^{\mu_{p}}$ for some infinite cardinal $\mu_{p} \leq|G|$.

Proof. See [EkMe, Lemma XII 5.2].

Together with Lemma 2.3 and the next result due to Hiller, Huber and Shelah the characterization is complete if $\operatorname{Hom}(G, \mathbb{Z})=0$.

LEMma 2.8. For any cardinal $\nu_{0}$ of the form $\nu_{0}=2^{\mu_{0}}$ for some infinite $\mu_{0}$ and any sequence $\left(\nu_{p}: p \in \Pi\right)$ of cardinals less than or equal to $\nu_{0}$ such that each $\nu_{p}$ is either finite or of the form $2^{\mu_{p}}$ for some infinite $\mu_{p}$ there is a torsion-free group $G$ such that $\operatorname{Hom}(G, \mathbb{Z})=0$ and $r_{0}^{\mathrm{e}}(G)=\nu_{0}, r_{p}^{\mathrm{e}}(G)=\nu_{p}$ for all primes $p \in \Pi$.

Proof. See [HiHuSh, Theorem 3(b)]. 
3. The singular case. In this section we prove our main theorem which completes the characterization of $\operatorname{Ext}(G, \mathbb{Z})$ for torsion-free groups $G$ under the assumption of Gödel's axiom of constructibility plus there is no weakly compact cardinal. The idea of the proof is as follows: For a singular cardinal $\nu$ of uncountable cofinality we shall construct a torsionfree abelian group $G$, of size $\nu$, as the union of pure subgroups $G_{\alpha}$ such that $G$ has prescribed values for $r_{0}^{\mathrm{e}}(G)$ and $r_{p}^{\mathrm{e}}(G)(p \in \Pi)$. Together with the $G_{\alpha}$ 's we also build homomorphisms $f_{\gamma}^{p}$ for $\gamma<r_{p}^{\mathrm{e}}(G)$ such that no non-trivial combination $\sum_{l<n} a_{l} f_{\gamma_{l}}^{p} \in \operatorname{Hom}(G, \mathbb{Z} / p \mathbb{Z})$ can be factored by the canonical homomorphism $\varphi^{p}: \operatorname{Hom}(G, \mathbb{Z}) \rightarrow \operatorname{Hom}(G, \mathbb{Z} / p \mathbb{Z})$ to a homomorphism in $\operatorname{Hom}(G, \mathbb{Z})$. This is a typical application of the diamond principle which holds under $(V=L)$ for every regular uncountable cardinal. On the other hand, we also need that for every $f \in \operatorname{Hom}(G, \mathbb{Z} / p \mathbb{Z})$ there are $f_{1}=\sum_{l<n} a_{l} f_{\gamma_{l}}^{p} \in \operatorname{Hom}(G, \mathbb{Z} / p \mathbb{Z})$ and $f_{2} \in \operatorname{Hom}(G, \mathbb{Z})$ such that $f-f_{1}=\varphi^{p}\left(f_{2}\right)$. The two demands seem to be hard to go together but the principle from [MeRoSh] allows us to carry out the construction.

THEOREM 3.1. $(V=L)$ Let $\nu$ be an uncountable singular cardinal of cofinality $\operatorname{cf}(\nu)>\aleph_{0}$ which is less than the first weakly compact cardinal. If $\left(\nu_{p}: p \in \Pi\right)$ is a sequence of cardinals less than or equal to $2^{\nu}$, then there exists a torsion-free group $G$ such that

(i) $|G|=\nu$;

(ii) $r_{0}^{\mathrm{e}}(G)=2^{\nu}$;

(iii) $r_{p}^{\mathrm{e}}(G)=\nu_{p}$ for all $p \in \Pi$.

Proof. First we note that by Lemma 2.8 for every prime $p \in \Pi$ there is a torsion-free group $G_{p}$ such that $\operatorname{Hom}\left(G_{p}, \mathbb{Z}\right)=0,\left|G_{p}\right|=\nu, r_{0}^{\mathrm{e}}\left(G_{p}\right)=2^{\nu}$ and $r_{p}^{\mathrm{e}}\left(G_{p}\right)=2^{\nu}=\nu^{+}$but $r_{q}^{e}\left(G_{p}\right)=0$ for all $q \neq p$. Since $\operatorname{Ext}(-, \mathbb{Z})$ commutes with direct sums it therefore suffices to assume $\nu_{p} \leq \nu$ for all $p \in \Pi$. Let $\kappa=\operatorname{cf}(\nu)$. Choose a continuous increasing sequence $\left\langle\mu_{\alpha}: \alpha<\kappa\right\rangle$ such that

- $\lim _{\alpha<\kappa} \mu_{\alpha}=\nu$;

- if $\alpha$ is a successor ordinal, then $\mu_{\alpha}=\lambda_{\alpha}^{+}$for some strong limit singular cardinal $\lambda_{\alpha}>\kappa$ such that $\operatorname{cf}\left(\lambda_{\alpha}\right)=\aleph_{0}$ and $\lambda_{\alpha+1}>\mu_{\alpha}$.

Now, let $S \subseteq\left\{\alpha<\kappa: \operatorname{cf}(\alpha)=\aleph_{0}\right\}$ be stationary. Inductively we shall construct a torsion-free group $G=\bigcup_{\alpha<\kappa} G_{\alpha}$ such that the following conditions are satisfied:

(i) $\left|G_{\alpha}\right|=\mu_{\alpha}$ and $G_{\alpha}$ is $\mu_{0}$-free; moreover, there is no $G^{\prime} \subseteq G_{\alpha}$ of cardinality less than $\mu_{\alpha}$ such that $G_{\alpha} / G^{\prime}$ is free.

(ii) $G_{\beta} \subseteq_{*} G_{\alpha}$ if $\beta<\alpha$.

(iii) $\operatorname{Hom}\left(G_{\alpha}, \mathbb{Z} / p \mathbb{Z}\right)=L_{p}^{\alpha} \oplus K_{p}^{\alpha}$ such that $f \uparrow_{G_{\alpha}} \in K_{p}^{\alpha}$ for all $f \in K_{p}^{\beta}$, $\alpha<\beta$. 
(iv) There are bases $B_{p}^{\alpha}$ of $K_{p}^{\alpha}$ as vector spaces over $\mathbb{Z} / p \mathbb{Z}$ and functions $T_{\alpha}: B_{p}^{\alpha} \rightarrow \operatorname{Hom}\left(G_{\alpha}, \mathbb{Z}\right)$ such that

(a) $f \uparrow_{G_{\alpha}} \in B_{p}^{\alpha}$ for all $f \in B_{p}^{\beta}, \alpha<\beta$;

(b) $\varphi^{p} T_{\alpha}=\operatorname{id}_{B_{p}^{\alpha}}$, where $\varphi^{p}$ is the canonical map $\varphi^{p}: \operatorname{Hom}\left(G_{\alpha}, \mathbb{Z}\right)$ $\rightarrow \operatorname{Hom}\left(G_{\alpha}, \mathbb{Z} / p \mathbb{Z}\right)$

(c) if $\alpha<\beta$ and $f \in B_{p}^{\beta}$, then $T_{\alpha}\left(f \uparrow_{G_{\alpha}}\right)=T_{\beta}(f)\left\lceil_{G_{\alpha}}\right.$.

(v) There are bases $\left\langle f_{\gamma}^{\alpha, p}: \gamma<\nu_{p} \cap \mu_{\alpha}\right\rangle$ of $L_{p}^{\alpha}$ and $M_{\alpha} \subseteq \operatorname{Hom}\left(G_{\alpha}, \mathbb{Z}\right)$ (for $\alpha \neq 0$ ) such that

(a) $f_{\gamma}^{\alpha, p} \subseteq f_{\gamma}^{\beta, p}$ for all $\gamma<\nu_{p} \cap \mu_{\alpha}, \alpha<\beta$;

(b) $M_{\alpha}=\bigoplus_{\gamma<\nu_{p} \cap \mu_{\alpha}, \delta \in[\alpha, \kappa)} \mathbb{Z} h_{\gamma, \delta}^{\alpha, p}$ and $\bar{p} h_{\gamma, \delta}^{\alpha, p}=f_{\gamma}^{\alpha, p}$ for all $\delta \in$ $[\alpha, \kappa)$, where $\bar{p}$ is the canonical map $\bar{p}: \mathbb{Z} \rightarrow \mathbb{Z} / p \mathbb{Z}$;

(c) for $\alpha<\beta \leq \delta<\kappa$ and $\gamma<\nu_{p} \cap \mu_{\alpha}$ we have $h_{\gamma, \delta}^{\alpha, p} \subseteq h_{\gamma, \delta}^{\beta, p}$;

(d) if $\alpha \in S$ and $g \in \operatorname{Hom}\left(G_{\alpha+1}, \mathbb{Z}\right)$ and $\bar{p} g \in L_{p}^{\alpha+1} \backslash\{0\}$, then $g \in M_{\alpha+1}$

(e) if $\nu_{p} \cap \mu_{\alpha} \leq \gamma<\nu_{p} \cap \mu_{\alpha+1}$, then $f_{\gamma}^{\alpha+1, p} \uparrow_{G_{\alpha}}=0$ and $h_{\gamma, \varrho}^{\alpha+1, p} \uparrow_{G_{\alpha}}=0$ for all $\varrho \in[\alpha+1, \kappa)$.

We first show that it is sufficient to carry out the inductive construction of $G_{\alpha}(\alpha<\kappa)$. Assume that the torsion-free groups $G_{\alpha}(\alpha<\kappa)$ are constructed satisfying conditions (i) to (v). Put $G=\bigcup_{\alpha<\kappa} G_{\alpha}$. Then $G$ is a torsion-free group of cardinality $\nu$. Moreover, $r_{0}^{\mathrm{e}}(G)=2^{\nu}$ follows from Lemma 2.3 and property (i). It remains to prove that $r_{p}^{\mathrm{e}}(G)=\nu_{p}$ for all $p \in \Pi$. Let $p \in \Pi$ and $\alpha<\nu_{p}$. We define

$$
f_{\alpha}^{\kappa, p}=\bigcup\left\{f_{\alpha}^{\gamma, p}: \gamma \in\left[\delta_{\alpha}, \kappa\right)\right\}
$$

where $\delta_{\alpha}=\min \left\{\delta<\kappa: \mu_{\delta} \geq \alpha\right\}$. By condition (v)(a) the function $f_{\alpha}^{\kappa, p}$ is a well defined homomorphism $f_{\alpha}^{\kappa, p} \in \operatorname{Hom}(G, \mathbb{Z} / p \mathbb{Z})$ for every $\alpha<\nu_{p}$. We shall show that

- $\left\{f_{\alpha}^{\kappa, p}: \alpha<\nu_{p}\right\}$ are linearly independent as elements of $\operatorname{Hom}(G, \mathbb{Z} / p \mathbb{Z})$;

- $\left\{f_{\alpha}^{\kappa, p}: \alpha<\nu_{p}\right\}$ are linearly independent in $\operatorname{Hom}(G, \mathbb{Z} / p \mathbb{Z})$ modulo $\operatorname{Hom}(G, \mathbb{Z}) \varphi^{p}$, i.e. no linear combination of them can be factored by $\bar{p}$ to a homomorphism from $G$ to $\mathbb{Z}$;

- $\left\{f_{\alpha}^{\kappa, p}: \alpha<\nu_{p}\right\}$ together with $\operatorname{Hom}(G, \mathbb{Z}) \varphi^{p}$ generate $\operatorname{Hom}(G, \mathbb{Z} / p \mathbb{Z})$.

Assume first that

$$
\sum_{\alpha \in E} z_{\alpha} f_{\alpha}^{\kappa, p}=0
$$


for some finite subset $E \subseteq \nu_{p}$ and elements $z_{\alpha} \in \mathbb{Z} / p \mathbb{Z}$. Then there exists $\beta$ such that $\alpha<\nu_{p} \cap \mu_{\beta}$ and $f_{\alpha}^{\beta, p}=f_{\alpha}^{\kappa, p} \Upsilon_{G_{\beta}} \neq 0$ for all $\alpha \in E$. Hence

$$
\sum_{\alpha \in E} z_{\alpha} f_{\alpha}^{\beta, p}=\left(\sum_{\alpha \in E} z_{\alpha} f_{\alpha}^{\kappa, p}\right) \uparrow_{G_{\beta}}=0 .
$$

But $\left\langle f_{\alpha}^{\beta, p}: \alpha<\nu_{p} \cap \mu_{\beta}\right\rangle$ is a basis of $L_{p}^{\beta}$ and thus $z_{\alpha}=0$ for all $\alpha \in E$. Therefore, the $f_{\alpha}^{\kappa, p}$, $\left(\alpha<\nu_{p}\right)$ are linearly independent.

Now, assume that there exists a finite linear combination $0 \neq \sum_{\alpha \in E} z_{\alpha} f_{\alpha}^{\kappa, p}$ which can be factored by $\bar{p}\left(0 \neq z_{\alpha} \in \mathbb{Z} / p \mathbb{Z}\right.$ for all $\left.\alpha \in E\right)$. Hence there is $0 \neq g \in \operatorname{Hom}(G, \mathbb{Z})$ such that

$$
\sum_{\alpha \in E} z_{\alpha} f_{\alpha}^{\kappa, p}=\bar{p} g
$$

Since $E$ is finite, there exists $\beta<\kappa$ such that $\alpha<\nu_{p} \cap \mu_{\beta}$ for all $\alpha \in E$. Therefore,

$$
\bar{p} g \uparrow_{G_{\gamma}}=\sum_{\alpha \in E} z_{\alpha} f_{\alpha}^{\kappa, p} \uparrow_{G_{\gamma}}=\sum_{\alpha \in E} z_{\alpha} f_{\alpha}^{\gamma, p}
$$

for every $\gamma \in[\beta, \kappa)$. By the linear independence of the $f_{\alpha}^{\kappa, p}$, s we may assume without loss of generality that $\bar{p} g \nwarrow_{G_{\gamma}} \neq 0$ for all $\gamma \in[\beta, \kappa)$ since otherwise we sufficiently enlarge $\beta$ so that $f_{\alpha}^{\kappa, p} \uparrow_{G_{\beta}} \neq 0$ for all $\alpha \in E$. We conclude that $\bar{p} g\left\lceil_{G_{\gamma+1}} \in L_{p}^{\gamma+1} \backslash\{0\}\right.$ for all $\gamma \geq \beta$ and condition (v)(d) implies that $g \uparrow_{G_{\gamma+1}} \in M_{\gamma+1}$ for all $\gamma \in S, \gamma \geq \beta$. Let $\delta=\gamma+1$ for some $\gamma \in S, \gamma \geq \beta$. Then

$$
g \uparrow_{G_{\delta}}=\sum_{k<k_{\delta}} b_{k}^{\delta} h_{\alpha_{k}^{\delta}, j_{k}^{\delta}}^{\delta, p}
$$

with $b_{k}^{\delta} \in \mathbb{Z} \backslash\{0\}$ and $\alpha_{k}^{\delta}<\nu_{p} \cap \mu_{\delta}, j_{k}^{\delta} \in[\delta, \kappa)$. Since the $h_{\beta, r}^{\delta, p}$ form a basis of $M_{\delta}$, this representation is unique.

By a pigeon hole argument we may assume that $k_{\delta}=k_{0}$ and $b_{k}^{\delta}=b_{k}$ for arbitrarily large $\delta=\gamma+1, \beta \leq \gamma \in S$. Let $\gamma_{0} \in S$ be sufficiently large such that $k_{0}=k_{\delta_{0}}$ with $\delta_{0}=\gamma_{0}+1$. Thus

$$
g\left\lceil_{G_{\delta_{0}}}=\left(g\left\lceil_{G_{\delta}}\right) \uparrow_{G_{\delta_{0}}}=\sum_{k<k_{0}} b_{k} h_{\alpha_{k}^{\delta_{0}, j_{k}} \delta_{0}}^{\delta_{0}}=\sum_{k<k_{0}} b_{k}\left(h_{\alpha_{k}^{\delta}, j_{k}^{\delta}}^{\delta, p} \uparrow_{G_{\delta_{0}}}\right)\right.\right.
$$

for all $\delta=\gamma+1, \gamma_{0} \leq \gamma \in S$. By condition (v)(e) and the two compatibility conditions $(\mathrm{v})(\mathrm{c})$ and $(\mathrm{v})(\mathrm{d})$ it easily follows that the following holds for all $\epsilon<\kappa$ :

$(\mathrm{v})\left(\mathrm{e}^{\prime}\right)$ if $\nu_{p} \cap \mu_{\alpha} \leq \gamma<\nu_{p} \cap \mu_{\epsilon+1}$, then $f_{\gamma}^{\epsilon+1, p} \uparrow_{G_{\alpha}}=0$ and $h_{\gamma, \varrho}^{\epsilon+1, p} \uparrow_{G_{\alpha}}=0$ for all $\varrho \in[\epsilon+1, \kappa)$.

Thus, if $\alpha_{k}^{\delta} \geq \nu_{p} \cap \mu_{\delta_{0}}$ (and also $\alpha_{k}^{\delta}<\nu_{p} \cap \mu_{\delta}$ ), then (v)( $\left.\mathrm{e}^{\prime}\right)$ implies that $\left.h_{\alpha_{k}^{\delta}, j_{k}^{\delta}}^{\delta, p}\right|_{G_{\delta_{0}}}=0$. Note that $\delta=\gamma+1$ for some $\gamma_{0} \leq \gamma \in S$. Hence $\alpha_{k}^{\delta}<\nu_{p} \cap \mu_{\delta_{0}}$ 
for all $k<k_{0}$ and therefore

$$
\left.h_{\alpha_{k}^{\delta}, j_{k}^{\delta}}^{\delta, p}\right\rceil_{\delta_{0}}=h_{\alpha_{k}^{\delta}, j_{k}^{\delta}}^{\delta_{0}, p}
$$

for all $j_{k}^{\delta}$. However, by uniqueness it follows that

$$
\left\{\alpha_{k}^{\delta_{0}}: k<k_{0}\right\}=\left\{\alpha_{k}^{\delta}: k<k_{0}\right\}
$$

and also

$$
\left\{j_{k}^{\delta_{0}}: k<k_{0}\right\}=\left\{j_{k}^{\delta}: k<k_{0}\right\}
$$

for all $\delta$ large enough. This contradicts the fact that $j_{k}^{\delta} \in[\delta, \kappa)$ for all $k<k_{0}$ and $\delta$. Therefore we obtain $\nu_{p} \leq r_{p}^{\mathrm{e}}(G)$ for all $p \in \Pi$.

It remains to prove that $r_{p}^{\mathrm{e}}(G)=\nu_{p}$ for all $p \in \Pi$. For this it suffices to show that $\left\langle f_{\alpha}^{\kappa, p}: \alpha<\nu_{p}\right\rangle$ generate $\operatorname{Hom}(G, \mathbb{Z} / p \mathbb{Z})$ modulo $\operatorname{Hom}(G, \mathbb{Z}) \varphi^{p}$. Hence, let $0 \neq g \in \operatorname{Hom}(G, \mathbb{Z} / p \mathbb{Z})$. We have to prove that there is a finite linear combination $\sum_{\alpha \in E} z_{\alpha} f_{\alpha}^{\kappa, p}$ with $E \subseteq \nu_{p}$ and $0 \neq z_{\alpha} \in \mathbb{Z} / p \mathbb{Z}$ such that

$$
g-\sum_{\alpha \in E} z_{\alpha} f_{\alpha}^{\kappa, p}=\bar{p} h
$$

for some $h \in \operatorname{Hom}(G, \mathbb{Z})$. Let $g_{\alpha}=g \uparrow_{G_{\alpha}}$ for all $\alpha<\kappa$. Hence, by (iii), there exist $k_{\alpha} \in K_{\alpha}^{p}$ and $l_{\alpha} \in L_{\alpha}^{p}$ such that $g_{\alpha}=k_{\alpha}+l_{\alpha}$ for every $\alpha<\kappa$ since $g_{\alpha} \in \operatorname{Hom}\left(G_{\alpha}, \mathbb{Z} / p \mathbb{Z}\right)$. Thus

$$
l_{\alpha}=\sum_{\beta \in E_{\alpha}} z_{\beta}^{\alpha} f_{\beta}^{\alpha, p}
$$

for some finite subset $E_{\alpha} \subseteq \nu_{p} \cap \mu_{\alpha}$ and $0 \neq z_{\beta}^{\alpha} \in \mathbb{Z} / p \mathbb{Z}$. Since this representation is unique we may assume by a pigeon hole argument that $z_{\beta}^{\alpha}=z_{\beta}$ and $E_{\alpha}=E$ are independent of $\alpha<\kappa$. Note that $f_{\beta}^{\alpha, p} \uparrow_{G_{\alpha^{\prime}}}=f_{\beta}^{\alpha^{\prime}, p}$ if $\alpha^{\prime}<\alpha$ and $\beta<\nu_{p} \cap \mu_{\alpha^{\prime}}$ and 0 otherwise by $(\mathrm{v})\left(\mathrm{e}^{\prime}\right)$. We conclude that

$$
\bar{h}=g-\sum_{\beta \in E} z_{\beta} f_{\beta}^{\kappa, p}
$$

satisfies $\bar{h} \uparrow_{G_{\alpha}}=k_{\alpha}$ for all $\alpha<\kappa$. Since $B_{p}^{\alpha}$ forms a basis of $K_{p}^{\alpha}$ for all $\alpha<\kappa$ there is a finite subset $F_{\alpha} \subseteq B_{p}^{\alpha}$ and $0 \neq w_{b}^{\alpha} \in \mathbb{Z} / p \mathbb{Z}$ for $b \in F_{\alpha}$ such that

$$
l_{\alpha}=\sum_{b \in F_{\alpha}} w_{b}^{\alpha} b
$$

for all $\alpha<\kappa$. Again, a pigeon hole argument allows us to assume that $w_{b}=$ $w_{b}^{\alpha}$ and $F=F_{\alpha}$ are independent of $\alpha$ by uniqueness. Note that $b \uparrow_{G_{\beta}} \in B_{p}^{\beta}$ if $b \in B_{p}^{\alpha}$ and $\alpha>\beta$. Putting

$$
h=\sum_{b \in F} w_{b} \bigcup_{\alpha<\kappa} T_{\alpha}(b)
$$


it follows that $h \in \operatorname{Hom}(G, \mathbb{Z})$ is well defined by (iv) and hence $\bar{p} h=\bar{h}$. Therefore, $g-\sum_{\alpha \in E} z_{\alpha} f_{\alpha}^{\kappa, p}=\bar{h}$ has a lifting to $h \in \operatorname{Hom}(G, \mathbb{Z})$. This finishes the proof and it remains to show that we can carry on the induction as claimed, i.e. we have to construct groups $G_{\alpha}(\alpha<\kappa)$ such that (i)-(v) are satisfied. We shall distinguish four cases.

CASE A: $\alpha=0$. Let $G_{0}=\bigoplus_{\mu_{0}} \mathbb{Z}$. Moreover, put $L_{p}^{0}=\{0\}$ and $K_{p}^{0}=$ $\operatorname{Hom}\left(G_{0}, \mathbb{Z} / p \mathbb{Z}\right)$. Since $G_{0}$ is free, the existence of $T_{0}$ is obvious and if we choose $M_{0}=\{0\}$, all conditions (i)-(v) are satisfied for $G_{0}$.

CASE B: $\alpha$ is a limit ordinal. Then we let $G_{\alpha}=\bigcup_{\beta<\alpha} G_{\beta}$. As before we define $\delta_{\gamma}=\min \left\{\delta<\alpha: \mu_{\delta} \geq \gamma\right\}$ for $\gamma<\nu_{p} \cap \mu_{\alpha}$ and let

$$
f_{\gamma}^{\alpha, p}=\bigcup\left\{f_{\gamma}^{\beta, p}: \delta_{\gamma} \leq \beta<\alpha\right\}
$$

and similarly for $\delta \in[\alpha, \kappa)$ and $\gamma<\nu_{p} \cap \mu_{\alpha}$ we let

$$
h_{\gamma, \delta}^{\alpha, p}=\bigcup\left\{h_{\gamma, \delta}^{\beta, p}: \delta_{\alpha} \leq \beta<\alpha\right\} .
$$

By the continuity conditions (v)(a) and $(\mathrm{v})(\mathrm{c})$ this is well defined. Hence, also $M_{\alpha} \subseteq \operatorname{Hom}\left(G_{\alpha}, \mathbb{Z}\right)$ and $L_{p}^{\alpha} \subseteq \operatorname{Hom}\left(G_{\alpha}, \mathbb{Z} / p \mathbb{Z}\right)$ are defined canonically. Finally, (iii) and the definition of $L_{p}^{\alpha}$ induce $K_{p}^{\alpha}$ as

$$
K_{p}^{\alpha}=\left\{f \in \operatorname{Hom}\left(G_{\alpha}, \mathbb{Z} / p \mathbb{Z}\right): f \uparrow_{G_{\beta}} \in K_{p}^{\beta} \text { for all } \beta<\alpha\right\} .
$$

The corresponding set $B_{p}^{\alpha}=\left\{f \in \operatorname{Hom}\left(G_{\alpha}, \mathbb{Z} / p \mathbb{Z}\right): f \uparrow_{G_{\beta}} \in B_{p}^{\beta}\right.$ for all $\left.\beta<\alpha\right\}$ is a basis for $K_{p}^{\alpha}$ and the continuity condition (iv)(c) allows us to define $T_{\alpha}=\bigcup_{\beta<\alpha} T_{\beta}$. It is easy to check that (i)-(v) are now satisfied.

CASE C: $\alpha=\beta+1$ and $\beta \notin S$. Then we let $G_{\alpha}=G_{\beta} \oplus \bigoplus_{\mu_{\alpha}} \mathbb{Z}$. In the obvious way we define $L_{p}^{\alpha}, K_{p}^{\alpha}, B_{p}^{\alpha}, T_{\alpha}, f_{\gamma}^{\alpha, p}$ for $\gamma<\nu_{p} \cap \mu_{\alpha}, M_{\alpha}$, and $h_{\gamma, \delta}^{\alpha, p}$ for $\gamma<\nu_{p} \cap \mu_{\alpha}$ and $\delta \in[\alpha, \kappa)$.

CASE D: $\alpha=\beta+1$ and $\beta \in S$. Here we imitate the proof of Main Theorem 3.9 of [MeRoSh]. We would like to avoid repeating the technical and lengthy construction from [MeRoSh] but instead point out the main changes for the convenience of the reader. It is then straightforward to modify the proof of Main Theorem 3.9 and its main ingredient Theorem 3.4 from [MeRoSh] and to adapt both to our setting.

We are in the following situation: $\lambda_{\alpha}$ is a strong limit singular cardinal strictly greater than $\kappa=\operatorname{cf}(\nu)$. Moreover, $\mu_{\alpha}=\lambda_{\alpha}^{+}=2^{\lambda_{\alpha}}$ is a regular cardinal, $\operatorname{cf}\left(\lambda_{\alpha}\right)=\aleph_{0}$ and $\lambda_{\alpha}=\lambda_{\beta+1}>\mu_{\beta}$ ( $\mu_{\alpha}$ plays the role of $\lambda$ in [MeRoSh], so it is the successor of a strong limit singular cardinal). Since we are assuming $(V=L)$ the prediction principle from [MeRoSh] holds. In Main Theorem 3.9 from [MeRoSh] it is proved that we can find a torsion-free group $\widetilde{G}$ (denoted by $G$ there) which has prescribed values $\widetilde{\nu}_{p}$ for $r_{p}^{\mathrm{e}}(\widetilde{G})$. The construction is very similar to ours, i.e. homomorphisms $\widetilde{f}_{\gamma}^{p} \in \operatorname{Hom}(\widetilde{G}, \mathbb{Z} / p \mathbb{Z})\left(\gamma<\widetilde{\nu}_{p}\right)$ are 
constructed (denoted by $f_{\lambda}^{p, \xi}$ there) which cannot be factorized by $\bar{p}$ (see the proof of Main Theorem 3.9 in [MeRoSh, p. 346]). The main tool is Theorem 3.4 from [MeRoSh] which can be seen as a Step Lemma since it deals with the "killing" of only one undesired homomorphism. However, we are at stage $\alpha$ of our construction, hence we do not want that our homomorphisms $f_{\gamma}^{\beta, p}\left(\gamma<\nu_{p} \cap \mu_{\beta}\right)$ which we have dealt with so far have no extension to $G_{\alpha}$, i.e. cannot be factorized by $\bar{p}$, but we just require that there are only some extensions, namely a set of extensions of $f_{\gamma}^{\alpha, p}$ which is assigned to each $f_{\gamma}^{\alpha, p}$. The role of this set is played by $\left\{h_{\gamma, \delta}^{\alpha, p}: \delta \in[\alpha, \kappa)\right\}$ (see (v)(b)). Thus the proof of Theorem 3.4 from [MeRoSh] carries over to our situation (for the case of successor cardinal of a strong limit singular cardinal of cofinality $\aleph_{0}$ ). Since the role of $\lambda$ in [MeRoSh, Theorem 3.4] is played by $\mu_{\alpha}$ in our setting only cases $\mathrm{B}$ and $\mathrm{C}$ in the proof of Theorem 3.4 remain. As usual one guesses the undesirable factorizations and kills them without affecting the work towards lifting that has already been done. The only difference is that we do not require that there is no lifting but we allow only the assigned ones. Now the adjusted version of Theorem 3.4 from [MeRoSh] is used in Main Theorem 3.9 from [MeRoSh] in the case of $\lambda$ being a successor of a strong limit cardinal. The resulting group serves as our $G_{\alpha}$.

REMARK 3.2. We would like to remark that the only reason for the choice of $\mu_{\alpha}$ as successor of a strong limit singular cardinal of cofinality $\aleph_{0}$ (if $\alpha$ is a successor ordinal) is that this is the easiest situation in the proof of [MeRoSh, Main Theorem 3.9]. However, the strategy described in Case D of the above proof of Theorem 3.1 (i.e. not killing all extensions of a homomorphism but allowing some of them to survive) works for every regular uncountable cardinal which is not weakly compact, e.g. $\aleph_{1}$. For instance it follows easily for $\aleph_{1}$ from [EkMe, Theorem XII 4.10] using [EkMe, Lemmas XII 4.8 and XII 4.9].

\section{References}

[EkHu] P. C. Eklof and M. Huber, On the rank of Ext, Math. Z. 174 (1980), 159-185. [EkMe] P. C. Eklof and A. Mekler, Almost Free Modules. Set-Theoretic Methods, rev. ed., North-Holland Math. Library 65, North-Holland, Amsterdam, 2002.

[EkSh] P. C. Eklof and S. Shelah, The structure of $\operatorname{Ext}(A, \mathbb{Z})$ and $G C H$ : possible co-Moore spaces, Math. Z. 239 (2002), 143-157.

[Fu] L. Fuchs, Infinite Abelian Groups, Vols. I and II, Academic Press, 1970 and 1973.

[GrSh1] R. Grossberg and S. Shelah, On the structure of $\operatorname{Ext}_{p}(G, \mathbb{Z})$, J. Algebra 121 (1989), 117-128.

[GrSh2] - - - On cardinalities in quotients of inverse limits of groups, Math. Japonica 47 (1998), 189-197. 
[HiHuSh] H. Hiller, M. Huber and S. Shelah, The structure of $\operatorname{Ext}(A, \mathbb{Z})$ and $V=L$, Math. Z. 162 (1978), 39-50.

[Je] T. Jech, Set Theory, Academic Press, New York, 1973.

[Ku] K. Kunen, Set Theory. An Introduction to Independence Proofs, Stud. Logic Found. Math. 102, North-Holland, 1980.

[MeRoSh] A. Mekler, A. Rosłanowski and S. Shelah, On the p-rank of Ext, Israel J. Math. 112 (1999), 327-356.

[SaSh1] G. Sageev and S. Shelah, Weak compactness and the structure of $\operatorname{Ext}(G, \mathbb{Z})$, in: Abelian Group Theory (Oberwolfach, 1981), R. Göbel and A. E. Walker (eds.), Lecture Notes in Math. 874, Springer, 1981, 87-92.

[SaSh2] - - - On the structure of $\operatorname{Ext}(A, \mathbb{Z})$ in $Z F C^{+}$, J. Symbolic Logic 50 (1985), 302-315.

[Sh1] S. Shelah, Whitehead groups may not be free even assuming $C H$, I, Israel J. Math. 28 (1977), 193-203.

[Sh2] - Whitehead groups may not be free even assuming CH, II, ibid. 35 (1980), $257-285$.

[Sh3] - The consistency of $\operatorname{Ext}(G, \mathbb{Z})=\mathbb{Q}$, ibid. 39 (1981), 74-82.

Department of Mathematics

The Hebrew University of Jerusalem

Jerusalem 91904, Israel

E-mail: shelah@math.huji.ac.il

and

Rutgers University

New Brunswick, NJ 08903, U.S.A.
Department of Mathematics University of Duisburg-Essen 45117 Essen, Germany E-mail: lutz.strungmann@uni-due.de

Current address of L. Strüngmann: Department of Mathematics University of Hawaii 2565 McCarthy Mall Honolulu, HI 96822-2273, U.S.A. E-mail: lutz@math.hawaii.edu

Received 22 December 2005;

in revised form 19 September 2006 